\title{
EDITORIAL
}

\section{Cortical noradrenaline, attention and arousal $^{1}$}

Since Marthe Vogt discovered the presence of noradrenaline (NA) in the central nervous system in the mid 1950s, this brain neurotransmitter has been implicated in a plethora of functions, ranging from the control of blood pressure to neuronal plasticity, memory and learning. Most interest has focused on the so-called dorsal noradrenergic ascending bundle (DNAB) which arises from cell bodies in the locus coeruleus of the dorsal pons. Utilizing novel histochemical techniques, Swedish research workers demonstrated that this nucleus not only had descending projections to the spinal cord and cerebellum, but also ascending projections to cortical areas such as the neocortex and hippocampus (Dahlström \& Fuxe, 1964; Ungerstedt, 1971). It is the latter projections especially which have captured the imagination of neuroscientists and will be the main subject of this brief review. Part of the reason why this 'veteran' neurotransmitter substance is still engendering a good deal of interest, even in the vanguard of the neuropeptides, is that genuine advances are emerging in the understanding of its possible roles in brain function. Another reason is that many psychoactive drugs are known to modulate activity of this ascending NA system. For example, opiates such as morphine, and antidepressants such as desipramine, reduce the activity of coeruleal NA neurones, although through different receptor mechanisms (see Olpé et al. 1983). In addition, central NA mechanisms are thought to modulate the behavioural effects of peptides such as arginine vasopressin, which has been used to treat human memory disorders (Kovacs et al. 1979). Indeed, changes in DNAB activity have been directly implicated both in Alzheimer's disease and in Korsakoff's psychosis (see Crow, 1981).

One consequence of the likely clinical relevance of studying central NA mechanisms has been the continuing emergence of a number of sophisticated neuropharmacological tools for analysing further their nature and functions. Thus, there are now available NA neurotoxins such as 6-hydroxydopamine (6-OHDA) and DSP-4, as well as drugs which affect the pre-synaptic release and uptake of NA (e.g. amphetamine and imipramine) and a host of agonists and antagonists for adrenergic receptors of different types together with sensitive receptor assays. This sophistication has undoubtedly contributed in a major way to the advances to be described.

There is still a good deal we do not know about the basic neuroanatomical and neurophysiological features of nucleus locus coeruleus and the DNAB. For example, the nature of the afferents to locus coeruleus and the organization of the DNAB are still a matter of debate. Early findings suggested that there was little discrete organization of the various NA projections of locus coeruleus. For example, a single cell body could give rise to both ascending and descending axons. However, some recent evidence has suggested a degree of topographical organization of the projection so that certain spatially-grouped collections of cell bodies project predominantly to some forebrain areas, whereas other groups project elsewhere. Nevertheless, Morrison \& Magistretti (1983) have contrasted the rather diffuse innervation of neocortex by the DNAB with that of other cortical afferents.

\section{EARLY PROPOSALS FOR DNAB FUNCTION}

The peculiar features of organization of the DNAB suggested to some workers that it had a rather general function. Clearly, messages relayed to at least a fair proportion of NA cell bodies would result in impulses simultaneously being transmitted to widespread portions of brain as far afield as the cerebellum and neocortex. In functional terms this supported an early proposal by Jouvet

\footnotetext{
1 Address for correspondence: Dr Trevor W. Robbins, Department of Experimental Psychology, University of Cambridge, Downing Street, Cambridge CB2 3EB.
} 
(1974), among others, that the DNAB was implicated in arousal: that is to say, a non-specific tonic state of neural activity which modulates not only the sleep/waking cycle, but also the efficiency of performance in the waking state. Arousal itself depended not only upon endogenous determinants but also upon the non-specific, intensive aspects of stimulus input including novelty, as opposed to its informational or 'cue' properties. Electrophysiological recordings from single units show activity in locus coeruleus cells to be highest during waking, at intermediate levels in slow-wave sleep, and at its lowest levels during REM sleep, an ordering which correlates with the behavioural degrees of arousal associated with these three states. However, there is also a good deal of evidence showing that the DNAB may not play an essential role in sleep regulation, and there is little doubt that a unidimensional concept of arousal is now in any case of little explanatory value. For example, Eysenck (1982), in a review of the evidence from experimental psychology, suggests that increasing arousal is linearly related to efficiency of certain aspects of performance except at very high levels which disrupt performance through anxiety, thereby generating the ubiquitous inverted U-shaped function relating arousal level to performance.

Other theorists had turned their attention to more specific functions of DNAB. It was known that rats would self-administer apparently rewarding trains of intracranial stimulation to those portions of brain rich in catecholamine-containing neurones, including of course the DNAB. Stein (1968) proposed that NA was the reward neurotransmitter, whereas Crow (1968) and Kety (1970) seized on the possibility that cortical NA provided the necessary reinforcing impact for learning to occur. Although early results involving electrolytic destruction of locus coeruleus supported this interpretation, later lesions produced by 6-OHDA resulting in more selective and profound loss of cortical NA failed to alter acquisition in the rat, at least of simple associations such as learning to press a lever for food on a continuously reinforced basis, or of learned taste aversion (Mason \& Iversen, 1979).

\section{DNAB AND NEURAL PLASTICITY}

Notwithstanding the apparent lack of involvement in learning per se, some remarkable effects of central NA mechanisms have been shown on synaptic plasticity in response to altered environmental input, particularly in development. For example, Kasamatsu and colleagues (for a review, see Kasamatsu, 1983) have employed the well-known model of binocularity in cat visual cortical cells to investigate the possible role of the neocortical NA innervation in the changes in ocular dominance produced by monocular deprivation during the post-natal critical period for the development of binocular cells. Noradrenergic depletion produced by 6-OHDA injected either intraventricularly, or by a sophisticated method of local perfusion of cortical zones, reduced the plasticity of visual cortical cells following monocular deprivation: that is to say, ocular dominance of these cells failed to develop fully. In contrast, the ocular dominance could be restored by infusion of exogenous NA in a dose-related manner. Moreover, Kasamatsu found that the recovery of binocular vision (i.e. the reversion from ocular dominance) which followed only brief periods of monocular deprivation could be retarded by NA depletion and accelerated by infusions of NA itself. Thus, the expected change in binocularity was opposite to that in the earlier studies and so represented a general change in plasticity rather than a particular effect on ocularity. Infusion of the $\beta$-adrenergic receptor blocker propanolol, but not $\alpha$-adrenergic blockers, could mimic the effects of 6-OHDA, suggesting that this change in plasticity was mediated by $\beta$ receptors. Furthermore, the involvement of $\beta$ rather than $\alpha$ receptors makes it likely that the effects on plasticity are not mediated via some effects of the DNAB on the regulation of cerebral blood flow.

Using a completely different, more behavioural model, Sutherland et al. (1982) have obtained analogous results. They found that damage to the medial cortex in rats resulted in impaired acquisition of a spatial task, but only if the lesions were made when the rat was adult. If instead the brain damage was inflicted early in development, then the animals were able to recover from its effects and exhibit normal spatial learning. This behavioural recovery, however, was prevented by the intraventricular administration of 6-OHDA to the neonatal rats. Therefore, this set of findings again suggests that NA affects cortical plasticity, especially in the developmental stage. 
Although these findings give the impression that cortical NA has special functions in the development of neural connections early in life, this may be misleading. After all, animals are equipped with the DNAB for the whole of their lives; indeed, it is of interest that depletion of cortical NA appears to be implicated as a correlate of pre-senile dementia. Moreover, there are examples of apparent changes in plasticity, in electrophysiological terms, within hippocampus produced by DNAB destruction in adult rats (e.g. Bliss et al. 1981). In addition, Kasamatsu and colleagues themselves elegantly showed that plasticity in adult rats could be altered by central manipulations of cortical NA. Even more recently Keverne \& de la Riva (1982) have shown that a 'short term olfactory memory' manifested in endocrine terms as the 'Bruce effect' in adult mice can be abolished by 6-OHDA depletion of NA from the olfactory bulb. In the Bruce effect the blockade of pregnancy occurs only by an odour from a strange male mouse while in the NA-depleted females the response also occurs by the odour of the stud male. Thus, the noradrenergic mechanism apparently sets into motion a relatively short-term olfactory memory which protects the female from the pregnancy block which would otherwise be induced by her stud male.

Most of these studies do not specify the nature of the conditions or stimuli producing activity in NA-containing cells. It seems most unlikely that NA is simply a modulatory neurohumour unresponsive itself to environmental conditions. Presumably, its effects on plasticity are normally mediated by induced activity in central NA neurones. How specific are the conditions producing such activity? For example, in the case of the olfactory memory described above, it is possible that cervical stimulation may be the trigger which increases NA turnover and thus facilitates 'memory'formation in the olfactory bulb (E. B. Keverne, personal communication). However, evidence described below suggests that the central NA mechanisms may be influenced by far more general classes of stimuli. In that case, the specificity of the effect of 6-OHDA lesions of olfactory bulb (and visual cortex in the case of Kasamatsu's experiments) may depend on the specificity of the neurones within the cortical areas to which the ascending NA neurones project.

In summary, these experiments have provided a confirmation of the Crow and Kety propositions that cortical NA modulates the long-term effects of experience on cortical function, although not to the extent of demonstrating definitive alterations in learning processes resulting from the changing impact of reinforcement conveyed by the ascending DNAB neurones.

\section{DNAB, ANXIETY AND STRESS}

An alternative, but no less specific, theory of dorsal bundle function was that activity within it was produced by stress, including, for example, anxiety produced by signals of punishment or non-reward. Gray (e.g. 1982) has argued that one of the means by which the minor tranquillizers (e.g. benzodiazepines) alleviate anxiety is by reducing activity in the DNAB. A related but thorny issue is the likely involvement of the DNAB in the phenomena related to depression. One well-known animal model of depression - learned helplessness - has long been associated with NA depletion, particularly in the coeruleal NA system (see Weiss et al. 1982). This depletion might be seen to result from a failure to replete the central NA system following the large increases in turnover produced by stressors such as unpredictable, inescapable electric shocks.

Previously, this NA depletion has been linked with the behavioural hypoactivity accompanying learned helplessness, although some workers have claimed that this hypoactivity is ancillary to a primary cognitive impairment in that model (see Gray, 1982, ch. 12 for a review). However, given the likely involvement of cortical NA in syndromes such as Alzheimer's disease and Korsakoff's psychosis, it seems quite possible that cortical NA depletion would also lead to cognitive impairment, especially in view of the types of deficit produced by DNAB lesions in animals. These deficits, which are described below, do not include behavioural hypoactivity, and infusions of low doses of NA into dense projections of the DNAB, such as the dentate gyrus of the hippocampus, do not produce the opposite effect of behavioural hyperactivity (Flicker \& Geyer, 1982). Therefore, the intriguing link between the motor retardation of the learned helplessness model and the locus coeruleus may not involve its cortical projection. Although the locus coeruleus NA system may well respond in a unitary fashion to stress, the different effects of the latter on learning and memory and on motor 
performance, for example, may result from the diverse influence of the coeruleal NA system on a variety of terminal regions, subcortical as well as cortical, which are specialized for those different functions. Activity in the DNAB may then enable the organization of a coordinated response to stress over a range of different behavioural and physiological functions.

\section{ELECTROPHYSIOLOGICAL APPROACHES TO DNAB FUNCTION}

Although it is the case that stressors such as electric shock can elevate NA turnover in the DNAB, a similar change in turnover can be elicited by other stimuli, not obviously aversive or stressful, such as rewarding intracranial stimulation (for a review, see Robbins \& Everitt, 1982). This fact, together with observations that cells in locus coeruleus respond electrophysiologically to polymodal, non-noxious inputs (Aston-Jones \& Bloom, 1981), again raises the possibility that the DNAB mediates general arousal as a function of stimulus intensity, novelty and learned salience, but not of detailed information about stimulus characteristics. In support of this Watabe et al. (1982) have shown how visual input from a variety of sources converges onto locus coeruleal cells and how such cells may respond to novel light flashes, but not to specific stimuli such as spots or slits. Earlier, the arousal possibility was mentioned, but was thought not to explain satisfactorily the complex role of the DNAB in sleep nor to recognize the inadequacy of monolithic constructs of arousal. To these doubts can be added the evidence that DNAB lesions produce only transient effects on cortical arousal as measured by the EEG (see Robbins \& Everitt, 1982). However, cortical EEG under baseline conditions is a fairly crude measure of the synaptic operations in neocortex and it may be more useful to consider the participation of NA in the evoked single cell responses to specific sensory inputs.

Bloom (1979) has argued that one of the functions of the DNAB is to extend in temporal terms the impact of environmental events, thus presumably converting phasic events into longer term tonic influences. He discusses an 'enabling' or 'biasing' mode of operation of the DNAB which enhances the response of the target cell to its other afferent responses without altering it directly. These ideas are based on the pioneering observations of Segal \& Bloom (e.g. 1976) who demonstrated that the electrical stimulation of locus coeruleus or microiontophoresis of NA onto pyramidal cells of the hippocampus enhanced the inhibitory effect of an auditory tone on hippocampal firing or, alternatively, enhanced the excitatory effect of the same stimulus when predictive of food.

Foote et al. (1975) have also shown that microiontophoresis of NA onto cells in the auditory cortex of the squirrel monkey changed the pattern of firing to species-specific vocalizations. Noradrenaline generally inhibited evoked firing, but did so to a greater extent for the background firing rate. This and other observations have led to the ideas that exogenously applied NA increases the signal to noise ratio $(\mathrm{S} / \mathrm{N})$ of the evoked responses. Analogous effects have been found in somatosensory cortex (Waterhouse \& Woodward, 1980) and Kasamatsu \& Heggelund (1982), studying single cell responses in cat visual cortex to visual stimulation during iontophoresis of NA, also recently found that the increased $\mathrm{S} / \mathrm{N}$ notion could satisfactorily summarize findings of diverse excitatory, as well as inhibitory, responses to NA. Thus this effect of cortical NA extends over at least three sensory modalities and seems to enhance the effects of stimuli that are specific for the cortical region studied.

\section{THE DNAB AND SELECTIVE ATTENTION : RECENT FINDINGS}

Extrapolating these electrophysiological findings to behavioural function is a perilous step which may not yet be completely justified. On balance, it would seem sensible to place the effects in a functional context at some level. One obvious possibility is that the change in $S / N$ ratio reflects alterations in sensory processing. However, just as little evidence has been found of motor impairment following DNAB lesions, there has also been little suggestion so far of sensory impairment. Recent work by Everitt et al. (1983) and by Carli et al. (1983), using visual and auditory discrimination of temporal frequency and spatial localization of brief, dim visual stimuli, has 
revealed no impairments in performance of rats with DNAB lesions. Mason \& Iversen (1979), arguing initially from indirect evidence, suggested alternatively that DNAB-lesioned animals have instead an attentional inability to screen out irrelevant stimuli. This is an ingenious proposal, but it does depend crucially upon how an irrelevant stimulus is defined. The most straightforward definition is a familiar stimulus that is only randomly correlated with reward. However, one might also possibly include stimuli (of the same or different sensory modality) which are redundant in predicting reward and novel, distracting stimuli of potential relevance which are extraneous to a particular task.

In brief, there have been several tests of this hypothesis, with rather conflicting results. One of the most important papers supporting the attentional hypothesis is that of Mason \& Lin (1980). In this article, a number of paradigms, well-known to learning theorists, are used to assess the extent to which rats have learned not to attend to stimuli that have in the past been unpredictive of reward. When such stimuli are subsequently made relevant, this will be advantageous to a rat unable to ignore these formerly irrelevant stimuli and hence will lead to a faster acquisition of response to them. Thus, under certain conditions, better acquisition should be predicted in rats with DNAB lesions. Although this facilitation of acquisition has been seen in a few experiments, it has in general proved difficult to obtain. For example, some of our own work has used a discrimination task in which there are two modalities, visual and auditory, and the rat was rewarded with food for discriminating fast or slow frequency flashes (or bleeps) in one modality, while ignoring the fast or slow stimuli in the other. Then, the previously irrelevant dimension was made relevant and vice versa, so that the DNAB-lesioned rat should have a clear advantage in shifting attention. However, we found that, unfortunately, the DNAB-lesioned rats were slower to learn the basic task which would invalidate any interpretation of faster reversal on their part because of their different training experiences (Robbins $e t$ al. 1982) (in fact, they were not in any case faster to reverse). This result would still support an attentional hypothesis if there was no difference in learning between the DNAB and sham-operated animals in the simple discrimination with only one modality present, but we have also found an acquisition impairment under those conditions (Everitt et al. 1983). Most of the DNAB rats did eventually learn, but of course this is entirely consistent with a modulatory rather than an essential role of the DNAB on stimulus processing of the type discussed by Bloom (1979). Control experiments ruled out the possibility of sensory or motivational impairment. For example, if the rats were trained to criterion before surgery, then there was no significant behavioural decrement by the cortical NA depletion: the deficit was in acquisition, not performance. We were surprised to find such apparently clear-cut deficits in acquisition, given the previous evidence. However, the temporal discrimination task was a difficult one, taking many sessions to learn, and the task may have been sufficiently sensitive to show an impairment following the marked capacity for recovery of function that is known to follow damage to the DNAB. It is also possible that the deficit was more apparent because of some special feature of the task, such as reliance on discrimination of temporal frequency, which may be a particularly 'cortical' type of function. However, we have now noted several other recent reports of acquisition failure after DNAB lesions (see Robbins et al. 1982).

These recent findings force us to reconsider the 'attentional hypothesis' because, although it is entirely possible that the deficits result from an attentional failure (that is, the discriminative stimuli fail to gain access to central associative mechanisms), it is also possible that the deficits result from a direct impairment of associative processes, as mooted by Crow and Kety. In order to test further the attentional hypothesis, extra pressure must be placed on selective attentional mechanisms in a situation in which the rat cortically depleted of NA and the sham controls are initially at the same level of performance. One way of achieving this is to present the discriminative stimuli with a shorter interval between them than is usual and, indeed, this does impair the performance of the DNABlesioned rat more than its control counterpart (Everitt et al. 1983; Carli et al. 1983). The other main means of disturbing performance is to introduce distracting stimuli. Interpolation of distractors simultaneously with the relevant discriminanda leads surprisingly to little disruption of performance, 
even in DNAB-lesioned rats. However, we have recently found, employing a task requiring the localization of brief visual target stimuli randomly at one of five locations, that playing loud ( $85 \mathrm{~dB})$ bursts of white noise just prior to the onset of the target visual stimuli can significantly impair the accuracy of detection of the visual target in the DNAB-lesioned but not the sham control rats (Carli et al. 1983). The interpolated white noise has other effects on performance: it increases the likelihood of premature, inappropriate responses (i.e. it makes the rats adopt a riskier criterion for responding), and it significantly hastens long reaction times in response to the light. Thus, it would appear that the white noise in some way arouses or activates motor performance. However, both of these effects are equivalent in the sham controls and DNAB-lesioned rats and it is only in the accuracy of detection that the latter perform worse. This suggests a quite specific behavioural impairment, and we shall speculate on this below.

The extreme measures we took to demonstrate a significant differential effect of distraction on the DNAB-lesioned rats make the type of attentional deficit shown by these animals seem less likely to be one of ignoring stimuli only randomly correlated with reward. On the whole, the DNAB-lesioned rat seems able to do this perfectly well. Of course, it is possible, as with the deficits in acquisition we observed, that the relatively small (though significant) effects result because of neurochemical recovery processes occurring within the DNAB. This requires further investigation. In addition, it would be worthwhile to attempt to activate or depress the coeruleo-cortical NA pathway by more acute manipulations (e.g. Flicker \& Geyer, 1982) to gain a more complete picture of the role of the DNAB in these attentional situations.

\section{THE DNAB, ATTENTION AND AROUSAL}

In accounting for these behavioural results we have made use of comparisons between the human experimental literature on attentional changes resulting from the manipulation of different environmental conditions. For example, the 5-choice visual localization test described above is obviously similar to Leonard's 5-choice serial reaction task, which is frequently used to assess the effect of environmental conditions and change in arousal produced, for example, by white noise and various drugs in human subjects (see Eysenck, 1982).

In our version of the continuous performance test it is notable that the stimulant $d$-amphetamine produces similar effects to those of white noise in normal rats: that is, over a wide dose range it has no effects on accuracy but increases premature responding and hastens reactions. It is now generally thought that the majority of the behavioural effects of $d$-amphetamine are mediated by central dopaminergic rather than noradrenergic mechanisms (which may, however, have a modulatory role). Complementing this fact, we have shown that dopamine (DA) depletion from the nucleus accumbens produces a slower response but no change in accuracy (Robbins et al. 1982). Therefore, it seems likely that the central NA and DA systems have rather different roles in controlling performance on this task. When arousing distractors, such as white noise, induce impulsive responding and threaten to disrupt discriminative performance, activity in the DNAB may maintain accuracy. However, from these preliminary results, the central mesolimbic DA system seems to be implicated in activating responding, making it both more likely and faster in execution, but not necessarily also guiding its accuracy. A distinction may then be made between mediation of the effects of arousal by cortical NA, and activation of behaviour by central DA mechanisms. This distinction, which runs counter to old unitary notions of arousal, may be analogous to Broadbent's concept of 'upper' and 'lower' arousal mechanisms (Broadbent, 1971) in which the upper mechanism monitors performance under conditions of supra-optimal activation of the lower mechanism (produced, for example, by white noise) and helps to prevent the disruptive effects of arousal on discrimination. The DNAB may then normally contribute to the functioning of this upper mechanism by enhancing inhibitory effects and preventing the organism from becoming overdistractible (see Fig. 1). This would appear to be broadly consistent with the electrophysiological studies of the DNAB reviewed above and with the findings that stressful stimuli increase NA turnover. An obvious prediction of the model is that the DNAB does not in itself mediate stress, 


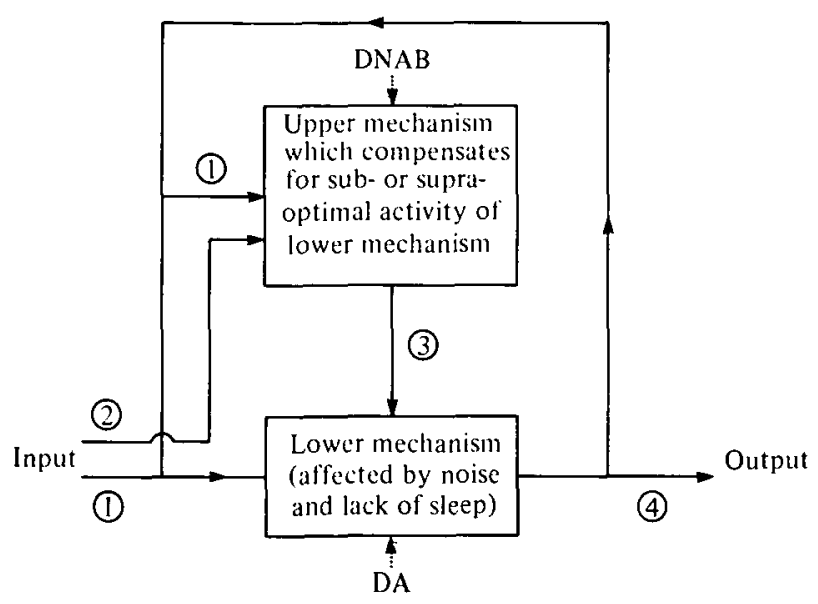

Fig. 1. Schematized diagram of the proposed 'upper' and 'lower' arousal mechanisms. Examples of the types of conditions influencing the lower mechanism are given on the figure. Stimulus input is hypothesized to have two main aspects: non-specific and arousing, produced by intense, or salient stimuli; and specific, involving informational properties such as spatio-temporal patterning. Both aspects are available to the upper mechanism, but only the arousing aspects to the lower mechanism. The latter controls primarily speed and probability of responding rather than response selection itself. The DNAB mediates arousal to the upper mechanism whereas the central DA systems may contribute to the activation of performance by the lower mechanism. For further description, see text. (1), non-specific arousal, drive (intensive aspects); (2), informational (discriminative aspects); (3), response selection or choice; (4), speed and likelihood of response.

but rather becomes active during stressful situations inducing high arousal, to preserve attentional selectivity. I would expect, in general, that impairments resulting from DNAB damage would most readily be seen under conditions of over-arousal of the lower mechanism, since the compensatory ability of the upper mechanism is lost. I would expect the lower mechanism to be aroused by salient arousing stimuli, including perhaps autonomic feedback, and to be mediated in part by activity in the central dopaminergic system. In contrast, the upper mechanism may also help to compensate for performance decrement produced by sub-optimal levels of arousal arising, for example, from lack of sleep. We can then perhaps explain why DNAB lesions in conjunction with adrenalectomy have such a devastating effect on avoidance performance in the rat (e.g. Ögren \& Fuxe, 1974) because, not only are the basic arousal processes of the animal deranged by adrenalectomy, but the upper mechanism which normally compensates for such deficiencies is also impaired.

There are many results which this speculative model probably cannot easily explain without extra assumptions. For example, in order to explain the selective effect of DNAB lesions on acquisition rather than performance, described above, it is necessary to assume that acquisition conditions involve higher levels of arousal than the conditions obtaining during the more automatic processes involved in performance. We would obviously have to predict, in parallel with this, elevated NA turnover in the DNAB during acquisition compared with performance.

Although I have emphasized the 'protective' effects of the DNAB on attention at elevated levels of arousal, it is also possible, given the possible exaggeration of effects of salient stimuli described by Segal \& Bloom (1976), that beneficial effects of arousal upon learning and memory are also mediated through the upper mechanism, as arousal apparently can improve long-term (though not short-term) memory in man (see Eysenck, 1982). We have no space here to review the effects of NA manipulations upon the consolidation of long-term memory traces, but there is some evidence that a reduction in central NA activity plays a central role in the effects of various amnesic agents such as electroconvulsive shock (Gold \& Sternberg, 1978). It is also possible that the apparently positive effects on memory of neuropeptides such as arginine vasopressin are also mediated by activity in the DNAB corresponding to enhanced levels of arousal, as Sahgal \& Wright (1983) have recently argued. 
I have tried to indicate how this account of DNAB function incorporates various hypotheses about DNAB function involving learning, arousal, stress and selective attention. The fact that it has not proved possible to provide conclusive evidence in favour of any one of these hypotheses may reflect their inter-relatedness, which I have tried to clarify. Furthermore, this more integrated account may explain how the relatively few neurones in locus coeruleus have been implicated in all of these functions.

A remaining puzzle is the relationship of the role of DNAB in arousal and in synaptic plasticity. Kasamatsu (1983) himself appears to believe that these dual functions are not directly related, although the DNAB influences each of them. However, there may be grounds for believing that changes in arousal can directly affect synaptic plasticity. For example, rearing animals in enriched environments can lead to definite changes in cortical synaptic development which some authors attribute to elevated arousal (Walsh \& Cummins, 1975). It is perhaps significant that the effects of $\mathrm{NA}$ on plasticity and on improving $\mathrm{S} / \mathrm{N}$ ratios both seem to involve the $\beta$ receptor, which is linked to adenylate cyclase, thus generating cyclic AMP as the second messenger. Cyclic AMP is known to be connected with intraneuronal metabolism, involving not only protein kinases that mediate phosphorylation, but also glycogenolysis and carbohydrate metabolism (see Morrison \& Magistretti, 1983), both of which may be implicated in neuronal plasticity. Therefore, the possibility arises that the metabolic and neuronal effects of cortical NA are quite closely related. This possibility would doubtless please Duffy, one of the earlier proponents of arousal as an important neural process, who believed that arousal or activation could be defined in terms of metabolic activity in the tissues: 'The level of activation of the organism may be defined as the extent of the release of potential energy, stored in the tissues of the organism, as this is shown in activity or response' (Duffy, 1962, p. 17). This bold statement may still have some relevance today, although we have come some way from the behavioural notion of arousal as Duffy intended, and we must not be distracted from specifying in much more detail the processes underlying this transduction.

T. W. ROBBINS

I thank Dr B. J. Sahakian and Dr E. B. Keverne for critically reading the manuscript and my collaborators, especially Dr B. J. Everitt, Dr M. Carli, Dr P. J. Fray and J. Evenden, for discussion. The author's recent experimental work upon which this editorial is based was supported by MRC grant G979/1150/N.

\section{REFERENCES}

Aston-Jones, G. \& Bloom, F. E. (1981). Norepinephrine-containing locus coeruleus neurones in behaving rats exhibit pronounced responses to non-noxious environmental stimuli. Journal of Neuroscience 1, 887-900.

Bliss, J. V. P., Goddard, G. V., Robertson, H. A. \& Sutherland, R. J.(1981). Noradrenaline depletion reduces long term potentiation in the rat hippocampus. In Cellular Analogues of Conditioning and Neural Plasticity (ed. O. Feher and F. Joo), pp. 175-185. Advances in Physiological Science Vol. 36. Pergamon Press: Oxford.

Bloom, F. (1979). Is there a neurotransmitter code in the brain? In Neurotransmitters (ed. P. Simon), pp. 205-213. Advances in Pharmacology and Therapeutics Vol. 2. Pergamon Press: Oxford and New York.

Broadbent, D E. (1971). Decision and Stress. Academic Press: New York.

Carli, M., Robbins, T. W., Evenden, J. \& Everitt, B. J. (1983). Effects of lesions to ascending noradrenergic neurons on performance of a 5-choice serial reaction task in rats: implications for theories of dorsal noradrenergic bundle function based on selective attention and arousal. Behavioural Brain Research 9, 361-380.

Crow, T. J. (1968). Cortical synapses and reinforcement. Nature 219, 736-737.

Crow, T. J. (1981). Biochemical aspects of memory. In Metabolic Disorders of the Nervous System (ed. F. Clifford Rose), pp. 369-375. Pitman: London.
Dahlström, A. \& Fuxe, K. (1964). Evidence for the existence of monoamine-containing neurons in the central nervous system. $I$. Demonstration of monoamines in the cell bodies of brain stem neurones. Acta Physiologica Scandinavica 62, Suppl. 232, 1-55.

Duffy, E. (1962). Activation and Behaviour. Wiley: London.

Everitt, B. J., Robbins, T. W., Gaskin, M. \& Fray, P. J. (1983). The effects of lesions to ascending noradrenergic neurones on discrimination learning and performance in the rat. Neuroscience 10, 397-410.

Eysenck, M. W. (1982). Attention and Arousal. Springer-Verlag: Berlin.

Flicker, C. \& Geyer, M. A. (1982). Behavior during hippocampal microinfusions, I. Norepinephrine and diversive exploration. Brain Research Reviews 4, 79-103.

Foote, S. L., Friedman, R. \& Oliver, A. P. (1975). Effects of putative neurotransmitters on neuronal activity in monkey cerebral cortex. Brain Research 86, 229-242.

Gold, P. E. \& Sternberg, D. B. (1978). Retrograde amnesia produced by several treatments; evidence for a common neurobiological mechanism. Science 201, 367-369.

Gray, J. A. (1982). The Neuropsychology of Anxiety. Oxford University Press: Oxford.

Jouvet, M. (1974). Monoaminergic regulation of the sleep-waking cycle in the cat. In The Neurosciences, Third Study Program (ed. F. O. Schmidt and F. G. Worden), pp. 499-508. MIT Press: Cambridge, Mass.

Kasamatsu, T. (1983). Neuronal plasticity maintained by the central norepinephrine system in the cat visual cortex. In Progress in 
Psychobiology and Physiological Psychology, Vol. 10 (ed. J. M. Sprague and A. N. Epstein), pp. 1-112. Academic Press: New York.

Kasamatsu, T. \& Heggelund, P. (1982). Single cell responses in cat visual cortex to visual stimulation during iontophoresis of noradrenaline. Experimental Brain Research 45, 317-324.

Kety, S.S. (1970). The biogenic amines and the central nervous system: their possible roles in arousal, emotion and learning. In The Neurosciences, Second Study Program (ed. F. O. Schmidt), pp. 324-336. Rockefeller University Press: New York.

Keverne, E. B. \& de la Riva, C. (1982). Pheromones in mice: reciprocal action between the nose and brain. Nature 296, 148-150.

Kovacs, G. L., Bohus, B. \& Versteeg, D. H. G. (1979). The effects of vasopressin on memory processes: the role of noradrenergic neurotransmission. Neuroscience 4, 1529-1537.

Mason, S. T. \& Iversen, S. D. (1979). Theories of dorsal bundle extinction effect. Brain Research Reviews 1, 107-137.

Mason, S. T. \& Lin, D. (1980). Dorsal noradrenergic bundle and selective attention. Journal of Comparative and Physiological Psychology 94, 819-832.

Morrison, J. H. \& Magistretti, P. J. (1983). Monoamines and peptides in cerebral cortex. Trends in Neuroscience 6, 146-151.

Ogren, S. \& Fuxe, K. (1974). Learning, noradrenaline and the pituitary-adrenal axis. Medical Biology 52, 399-405.

Olpé, H.-R., Jones, R. S. G. \& Steinmann, M. W. (1983). The locus cocruleus actions of psychoactive drugs. Experientia 39, 242-249.

Robbins, T. W. \& Everitt, B. J. (1982). Functional studies of the central catecholamines. International Review of Neurobiology 23, 303-365.

Robbins, T. W., Everitt, B. J., Fray, P. J., Gaskin, M., Carli, M. \& de la Riva, C. (1982). The roles of the central catecholamines in attention and learning. In Behavioral Models and the Analysis of Drug Action (ed. M. Y. Spiegelstein and A. Levy), pp. 109-134. Elsevier: Amsterdam.
Sahgal, A. \& Wright, C. (1983). A comparison of the effects of vasopressin and oxytocin with amphetamine and chlordiazepoxide on passive avoidance behaviour in rats. Psychopharmacology $\mathbf{8 0}$, 88-92.

Segal, M. \& Bloom, F. E. (1976). The action of norepinephrine in the rat hippocampus. IV: The effect of locus coeruleus stimulation on evoked hippocampal activity. Brain Research 107, 513-525.

Stein, L. (1968). Chemistry of reward and punishment. In Psychopharmacology: a Review of Progress 1957-1967 (ed. D. H. Efron), pp. 105-123. US Government Printing Office: Washington, D.C.

Sutherland, R. J., Kolb, B., Whishaw, I. Q. \& Becker, J. B. (1982). Cortical noradrenaline depletion eliminates sparing of spatial learning after neonatal frontal cortex damage in the rat. Neuroscience Letters 32, 125-130.

Ungerstedt, U. (1971). Stereotaxic mapping of the monoamine pathways in rat brain. Acta Physiologica Scandinavica 367 (Suppl.), $1-49$.

Walsh, R. N. \& Cummins, R. A. (1975). Mechanisms mediating the production of environmentally-produced brain changes. Psychological Bulletin 82, 986-1000.

Watabe, K., Nakai, K. \& Kasamatsu, T. (1982). Visual afferents to norepinephrine-containing neurones in cat locus coeruleus. Experimental Brain Research 48, 66-80.

Waterhouse, B. D. \& Woodward, D. J. (1980). Interaction of norepinephrine with cerebro-cortical activity evoked by stimulation of somatosensory afferent pathways. Experimental Neurology 67, 11-34.

Weiss, J. M., Bailey, W. H., Goodman, P. A., Hoffman, L. J., Ambrose, M. J., Salmon, S. \& Charry, J. M. (1982). A model for neurochemical study of depression. In Behavioral Models and the Analysis of Drug Action (ed. M. Y. Spiegelstein and A. Levy), pp. 195-233. Elsevier: Amsterdam. 